\title{
Novel Highly Porous Metal Technology in Artificial Hip and Knee Replacement: Processing Methodologies and Clinical Applications
}

\author{
JOHN MUTH, ${ }^{1}$ MATTHEW POGGIE,${ }^{1,3}$ GENE KULESHA, ${ }^{1}$ \\ and R. MICHAEL MENEGHINI ${ }^{2,4}$ \\ 1.-Stryker Orthopaedics, Mahwah, NJ 07430, USA. 2.-Department of Orthopaedic Surgery, \\ Indiana University School of Medicine, Indianapolis, IN 46202, USA. 3.-e-mail: matt.poggie@ \\ stryker.com. 4.—e-mail: rm_meneghini@yahoo.com
}

Hip and knee replacement can dramatically improve a patient's quality of life through pain relief and restored function. Fixation of hip and knee replacement implants to bone is critical to the success of the procedure. A variety of roughened surfaces and three-dimensional porous surfaces have been used to enhance biological fixation on orthopedic implants. Recently, highly porous metals have emerged as versatile biomaterials that may enhance fixation to bone and are suitable to a number of applications in hip and knee replacement surgery. This article provides an overview of several processes used to create these implant surfaces.

\section{FIXATION STRATEGIES WITH MODERN BIOMATERIALS}

Hip and knee replacement can dramatically improve a patient's quality of life through pain relief and restored function. Fixation of hip and knee replacement implants to bone is critical to the success of the procedure. A variety of roughened surfaces and three-dimensional (3-D) porous surfaces have been used to enhance biological fixation on orthopedic implants for over 30 years. ${ }^{1,2}$ More recently, highly porous metals have emerged as versatile biomaterials that may enhance fixation to bone and are suitable to a number of applications in hip and knee replacement surgery. ${ }^{3}$ The advantages provided by these newly developed porous metals may improve cementless fixation and long-term patient outcomes in hip and knee replacement.

\section{Historical Coating Technologies}

Thermal spray technologies involving the melting and subsequent spraying of metal feedstock have been leveraged by various implant manufacturers to apply highly roughened commercially pure titanium (CPTi) and titanium (Ti) alloy coatings onto implants used in hip and knee arthroplasty.

\section{Wire Arc Deposition}

Twin wire arc deposition is a traditional thermal spray technology that is used to deposit a highly rough and dense, two-dimensional coating onto acetabular shells and femoral hip stems used in hip arthroplasty. The technology employs a specially designed gun through which two CPTi sacrificial wires are fed. At the front of the gun, an electrical arc is struck between the tips of the two wires melting them as they are continuously driven into the arc. The resulting localized melt is then atomized by a high-pressure stream of argon gas, passed through the gun tip, causing the fine, molten titanium particles to be sprayed onto an implant held by specialized fixtures. The rough coating is continuously built up until a desired thickness of about 0.02 in is reached (Fig. 1).

The entire process is conducted in an inert environment of argon gas to ensure the CPTi coating is compliant with relevant chemistry specifications. The critical processing parameters of argon gun pressure, voltage, and amperage can be controlled to alter the degree of desired roughness and mechanical strength.

\section{Plasma Spray}

Plasma spray is another well-established process used by several orthopedic device manufacturers to 


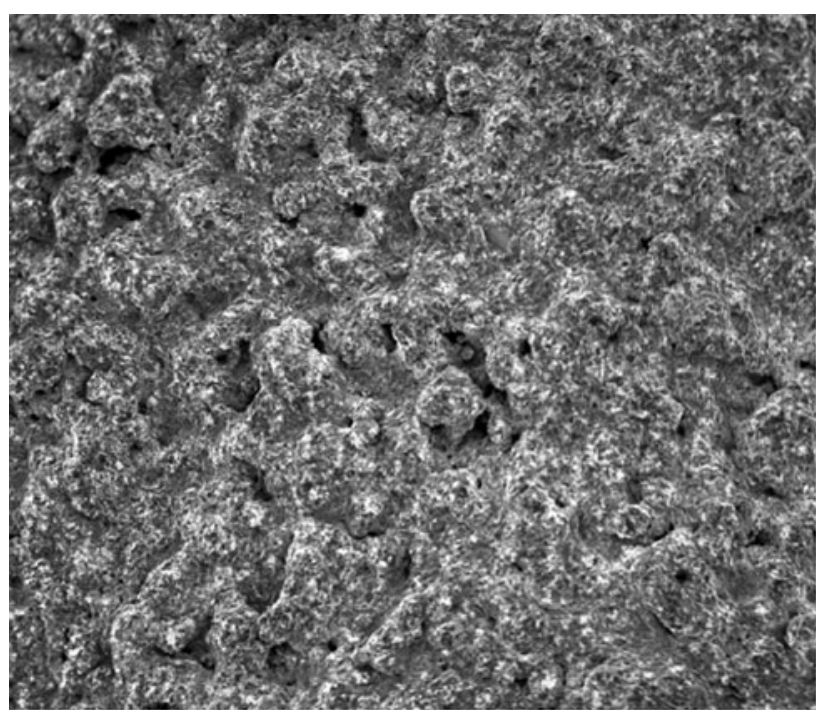

Fig. 1. Arc-deposited Ti, $\times 50$ magnification.

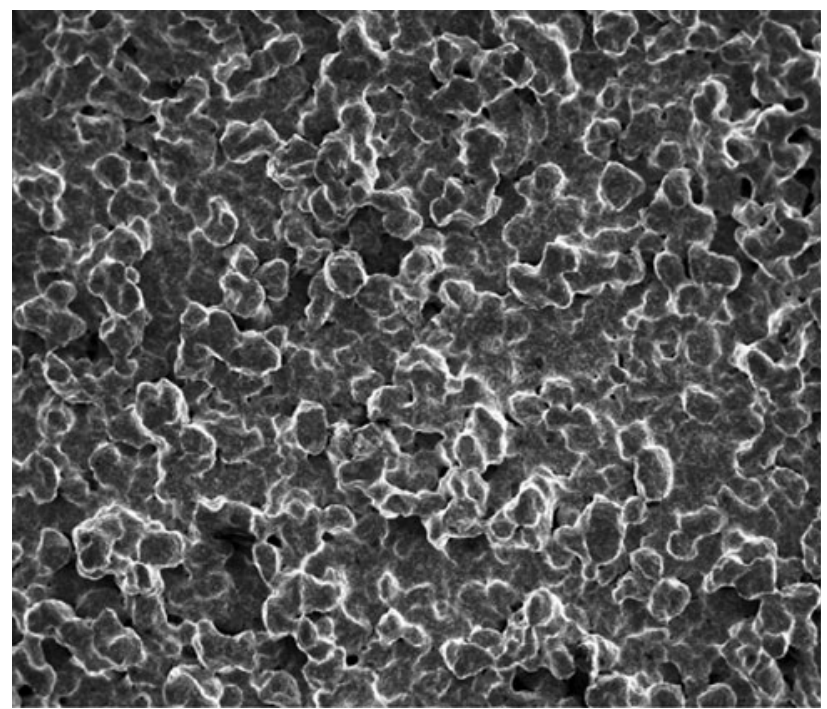

Fig. 2. Plasma-sprayed Ti, $\times 50$ magnification.

coat implants with highly roughened and dense Ti. Unlike the wire arc deposition process, the plasma spray process uses fine powder particles (10 $200 \mu \mathrm{m})$ as a feedstock and a plasma gun with a cathode and water-cooled anode, which are not sacrificial (as in the wire arc). The powder particles are continuously fed via a specialized hopper system into a plasma flame $\left(\sim 12,000^{\circ} \mathrm{C}\right)$, which is generated between the cathode and anode. After melting in the flame, the molten particles are propelled by the gun's high-pressure inert gas onto an implant where they essentially splat quench and rapidly solidify. After successive passes of the gun over the part, the solidified splats build up into a conformal coating (Fig. 2).

Coatings typically range from 0.01 to 0.03 in. Similar to wire arc, the plasma spray process is

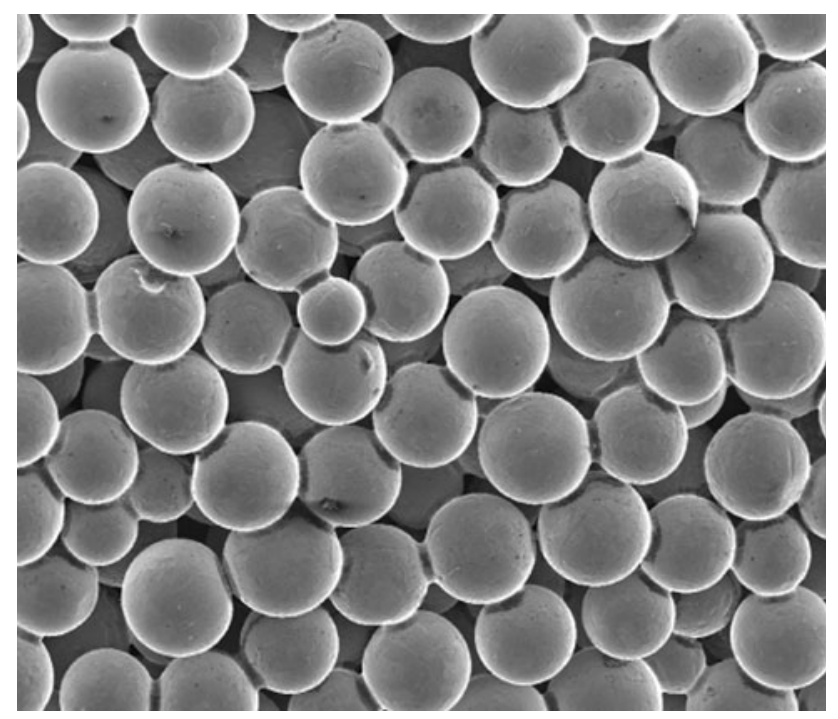

Fig. 3. Sintered CoCr beads, $\times 50$ magnification.

conducted within an inert argon atmosphere to avoid contamination of the titanium coatings. Processing parameters such as powder feedstock, gun pressure, amperage, and voltage can be altered to modify the physical and mechanical properties of the coating.

\section{Porous Beads}

Porous beaded surfaces have been developed and commercialized by several orthopedic device manufacturers. The starting feedstock material is made of a cobalt chromium molybdenum alloy conforming to ASTM F1377. The feedstock is in the form of spherical beads that are $500-700 \mu \mathrm{m}$ in diameter and are originally manufactured by a plasma rotating electrode process. The fabrication steps used to apply the beads to an implant begin with a manual process of applying an organic, watersoluble binder to the beads and an implant substrate's surface. Subsequently, the beads are attached to the implant via mechanical means where the binder causes the beads to adhere to each other and the receiving substrate. Following this "gluing" step, the bead-coated implants are subjected to a high-temperature sinter cycle in a vacuum furnace under inert conditions. The sintering process removes the binder and induces solid-state diffusion between the beads and between the beads and substrate implant (Fig. 3).

The bead application and sintering steps are repeated until a required coating thickness is achieved. Typical coating thicknesses are $1.5 \mathrm{~mm}$. Unlike wire arc and plasma spray coatings, which are two dimensional (i.e., pore free), the microstructured beaded coatings have a porosity of about $35 \%$ and an average pore size of $425 \mu \mathrm{m}$, which has been demonstrated to achieve biological fixation adequately between implants and host bone. ${ }^{4}$ 


\section{DIFFUSION-BONDED POROUS TITANIUM COATINGS}

Other methods have been employed to produce implant surfaces with a greater degree of porosity. The diffusion bonding process results in a solid solution between the surface compact and the underlying Ti alloy substrate, thus imparting good static and dynamic mechanical properties to the implant. Coating systems produced through this method uses CPTi, which has been demonstrated to exhibit osseointegration superior to $\mathrm{Ti}$ alloy in an animal model. ${ }^{5}$

In one method, CPTi wire is cut into predetermined lengths, compacted, and diffusion bonded to a Ti alloy implant substrate (Fig. 4). Because of the random nature of the wire-mesh structure, the pore size generally varies but overall porosity in the range of $50 \%$ can be achieved. ${ }^{6,7}$

Another process employs CPTi powder that is sintered to a $\mathrm{Ti}$ alloy substrate under high pressure and temperature. By virtue of the process, an interconnected network of pores is created that can yield coatings with a pore size in the range of $500 \mu \mathrm{m}$ and overall porosity near $55 \%{ }^{8}$ (Fig. 5).

\section{Advanced, Highly Porous Coating Technologies}

In an effort to increase the space available on the surface of an implant for biologic fixation manufacturers have developed processes to create highly porous surfaces using a variety of techniques.

Zimmer (Warsaw, IN) provides a highly porous surface for implant fixation that uses Tantalum as the biomaterial. ${ }^{9}$ This porous material is produced through the chemical vapor deposition/infiltration of tantalum onto a vitreous carbon core that was formed from the pyrolysis of a thermosetting polymer foam precursor. The material can be made into

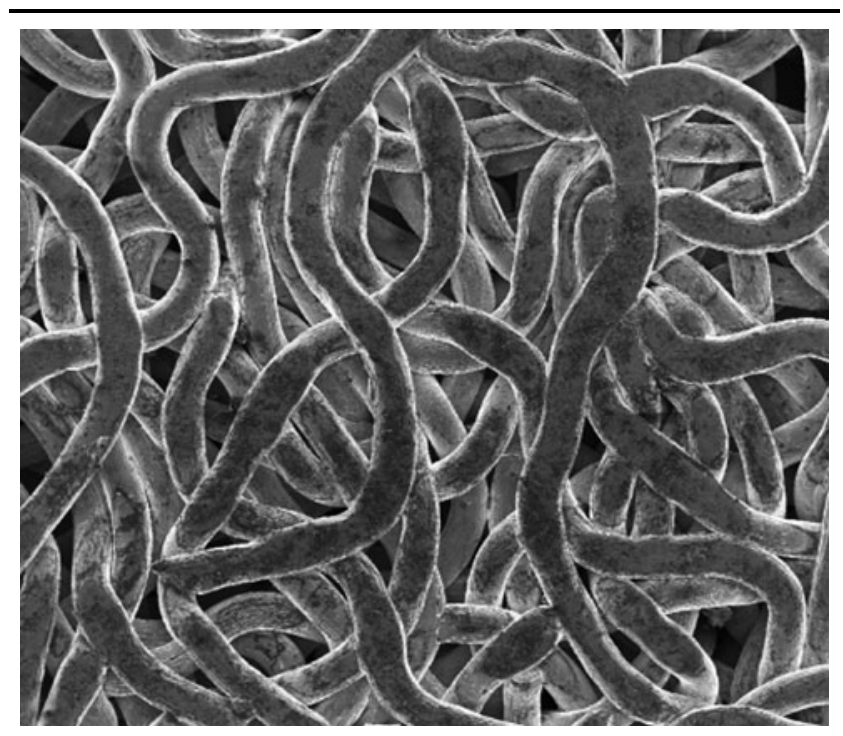

Fig. 4. Diffusion-bonded CPTi wire, $\times 50$ magnification. a bulk implant of specified size and shape or it can be used as a surface coating on a solid substrate. ${ }^{10}$ The structure of the porous surface mimics the morphology of the precursor and provides a regularly shaped and sized interconnected porosity (Fig. 6).

In addition to osseointegration and increased interface strength, ${ }^{10}$ porous tantalum provides the important biomechanical properties of increased material elasticity and a high surface coefficient of friction. The coefficient of friction for porous-tantalum on cancellous bone (0.88-0.98) is significantly greater than that previously reported for traditional porous-coated and sintered-bead materials (0.50$0.66){ }^{11}$ Furthermore, the modulus of elasticity of porous-tantalum is in between cortical and cancellous bone, significantly less than titanium and chromium cobalt materials. ${ }^{12}$ This optimal elasticity

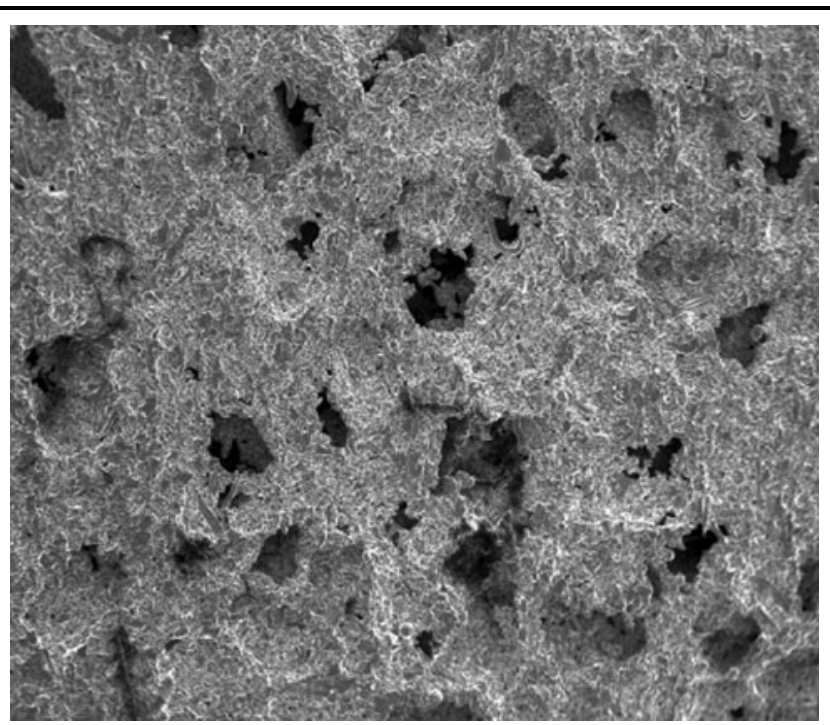

Fig. 5. Diffusion-bonded CPTi powder, $\times 50$ magnification.

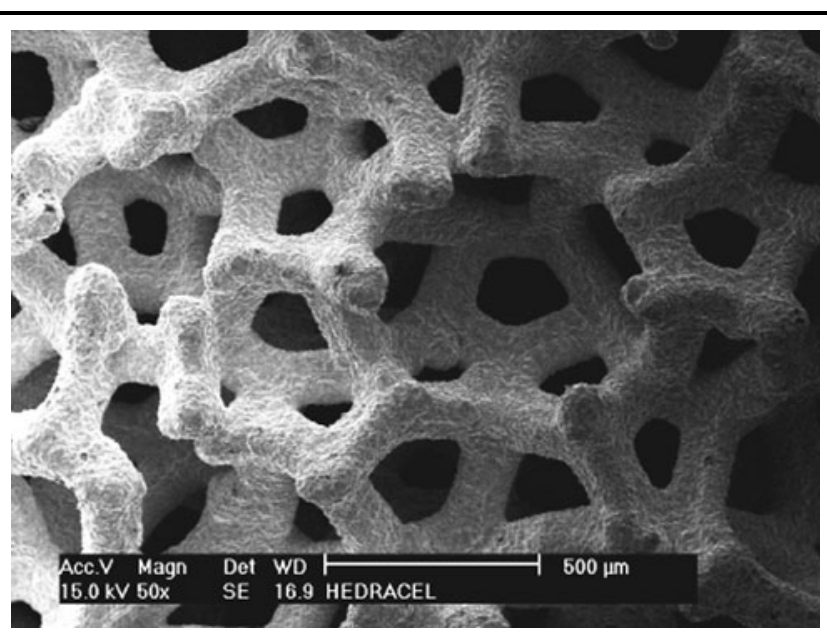

Fig. 6. Porous tantalum structure, $\times 50$ magnification. 
of porous tantalum may create a more physiologic transfer of stresses to the periprosthetic bone, theoretically decreasing detrimental stress shielding.

Highly porous titanium has also been developed to improve fixation strength to bone through a more biologically inspired macrostructure and microstructure. Stryker (Mahwah, NJ) has produced an open, porous 3-D surface using CPTi powder that is marketed under the name Tritanium.

The proprietary manufacturing process begins by machining a scaffold from a commercially available, fully reticulated, open cell, polyurethane foam to a desired form. The interconnecting pore structure of this sacrificial material provides the architecture for the resulting metallic structure (Fig. 7).

The machined foam is coated with a thin layer of CPTi that is applied via low-temperature arc vapor deposition. The CPTi coated polyurethane structure is placed on a solid $\mathrm{Ti}$ alloy substrate and the assembly is placed in a vacuum furnace. A sinter cycle is run whereby the polyurethane is volatilized and evacuated leaving the lightly sintered CPTi skin in the image of the polyurethane foam attached to the underlying Ti alloy substrate (Fig. 8).

A polymeric binder is then applied to the components. This binder is effective in adhering spherical CPTi powder that is applied to the 3-D scaffold. The component is once again exposed to a programmed sintering cycle. In the process, the binder is burned off and the Ti powders are sintered together as well as producing a firm attachment to the underlying $\mathrm{Ti}$ alloy substrate. Several powder coating and sintering operations are carried out in sequence until the desired interconnecting porous structure is obtained and the component is strengthened. This component can then be machined to a final implant design configuration (Fig. 9).

To get a closer look at the porous surface and to characterize the coating, scanning electron

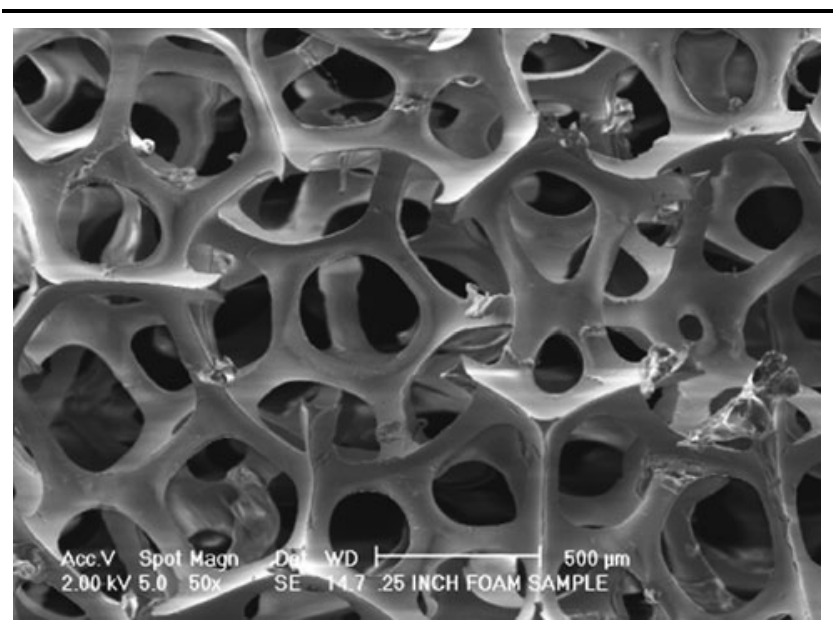

Fig. 7. Polyurethane foam. The complete cell unit has a 3-D shape in dodecahedron geometry. The larger pore is the inner dimension of the cross section of the cell and the smaller pores are the pentagonal shaped windows between adjacent cells, $\times 50$ magnification. microscopy, light microscopy, and metallographic techniques can be used. The magnified images of the actual porous $\mathrm{Ti}$ surface reveal an intricate architecture. This porous surface can be characterized as having a bimodal structure that is a combination of both larger "major pores" and smaller "interconnecting pores." The sintered powders create a web structure that defines the frame of the smaller interconnecting pore windows. These smaller windows composed of the solidified struts surround the larger major pores defining their ultimate dimension (Figs. 10, 11, and 12).

A cross section through the surface and substrate is taken and using quantitative image analysis techniques the depth and (vol.\% of voids) or (\% porosity) of the Tritanium porous surface can be determined (Fig. 13).

Using a different proprietary process, Stryker has produced an alternative porous $\mathrm{Ti}$ surface. This process employs the use of a sacrificial pore former, a polymeric binding agent, and an angular CPTi

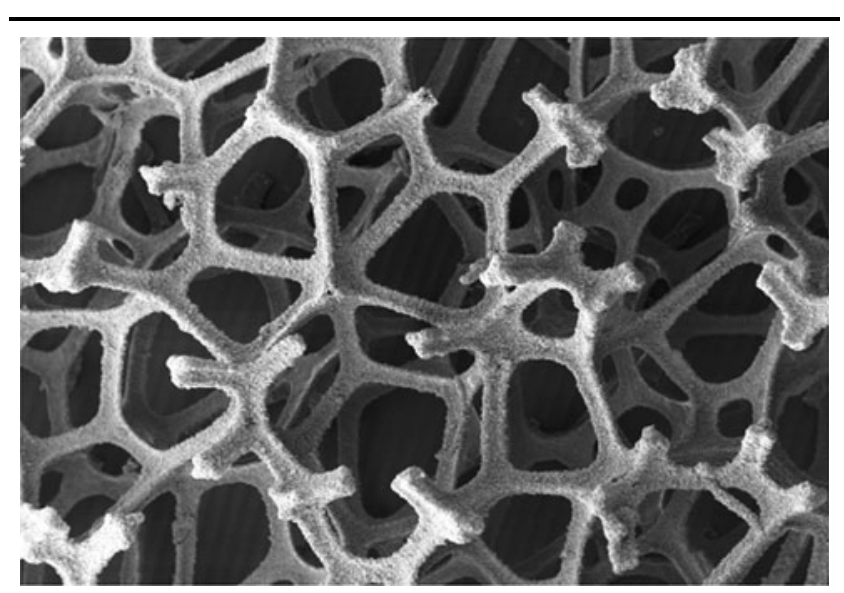

Fig. 8. Sintered CP Ti scaffold on underlying Ti alloy substrate, $\times 50$ magnification.

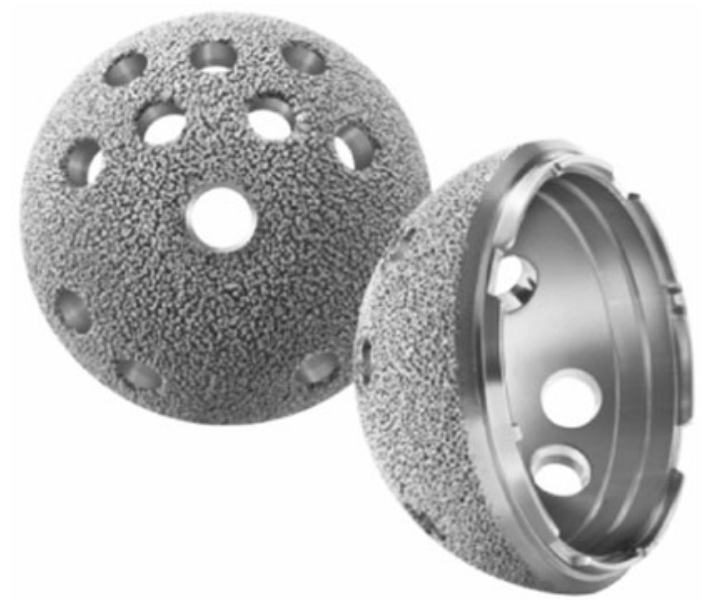

Fig. 9. Trident Tritanium acetabular shell, offered in outer diameter sizes ranging from 48 to $80 \mathrm{~mm}$ in diameter. 


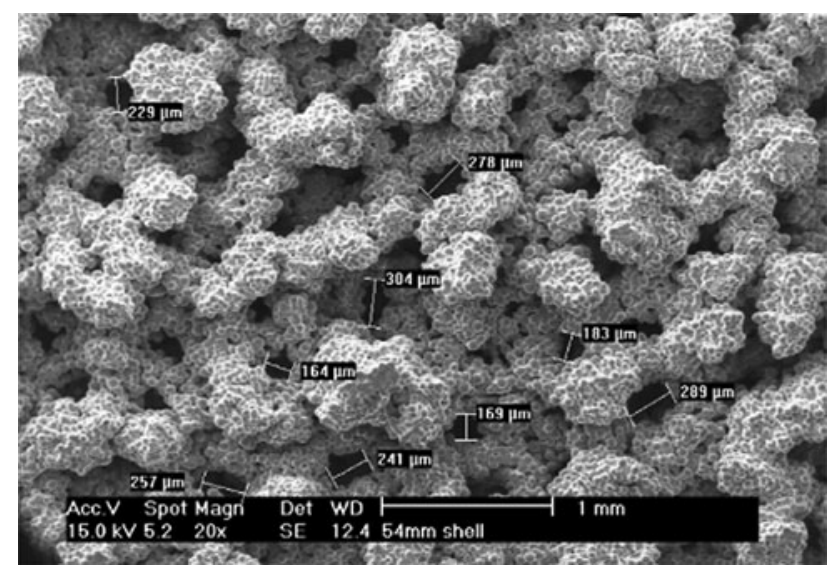

Fig. 10. Tritanium minor "interconnecting" pores, in the range of $250 \mu \mathrm{m}, \pm 100 \mu \mathrm{m}, \times 20$ magnification.

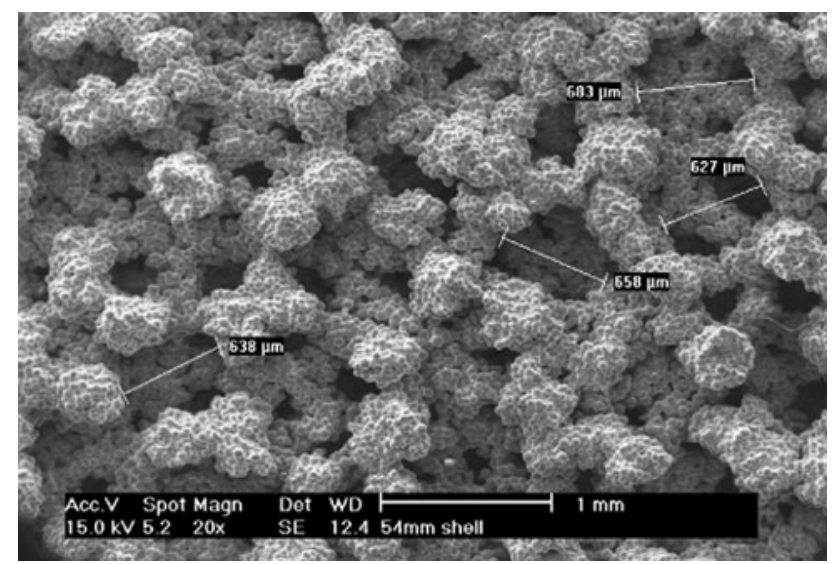

Fig. 11. Tritanium major pores, in the range of $650 \mu \mathrm{m}, \pm 100 \mu \mathrm{m}$, $\times 20$ magnification.

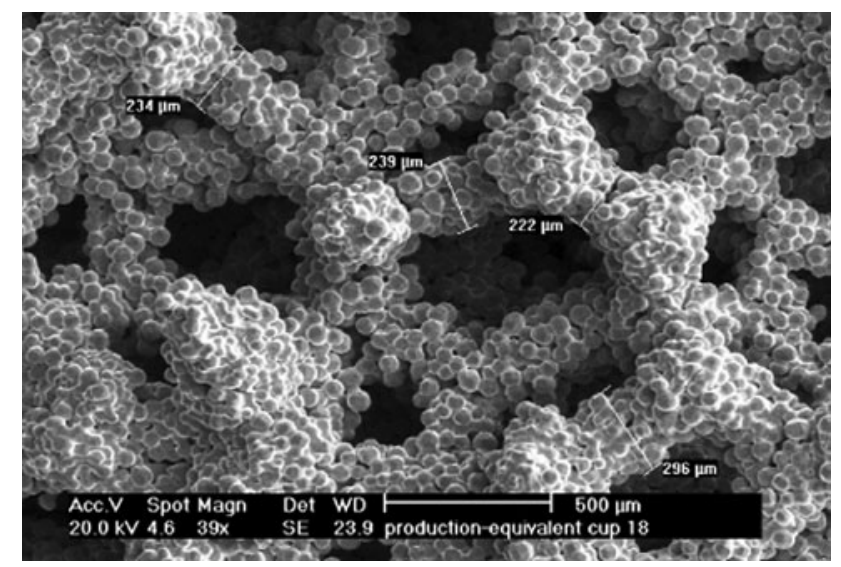

Fig. 12. Tritanium strut thickness, in the range of $235 \mu \mathrm{m}, \pm 33 \mu \mathrm{m}$, $\times 39$ magnification.

powder. These three components are blended together, transferred to a mold, then under high pressure at low temperature are compacted to form a "green state" structure. This green state component can be

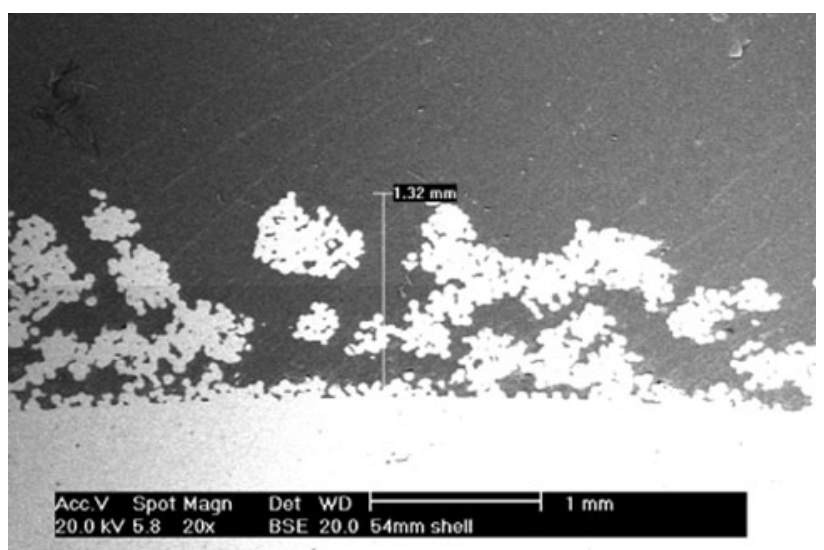

Fig. 13. Tritanium porous surface depth, in the range of $1.30 \mathrm{~mm} \pm 0.15 \mathrm{~mm}$. Porosity is approximately $65 \%$ across the component size range, $\times 20$ magnification.

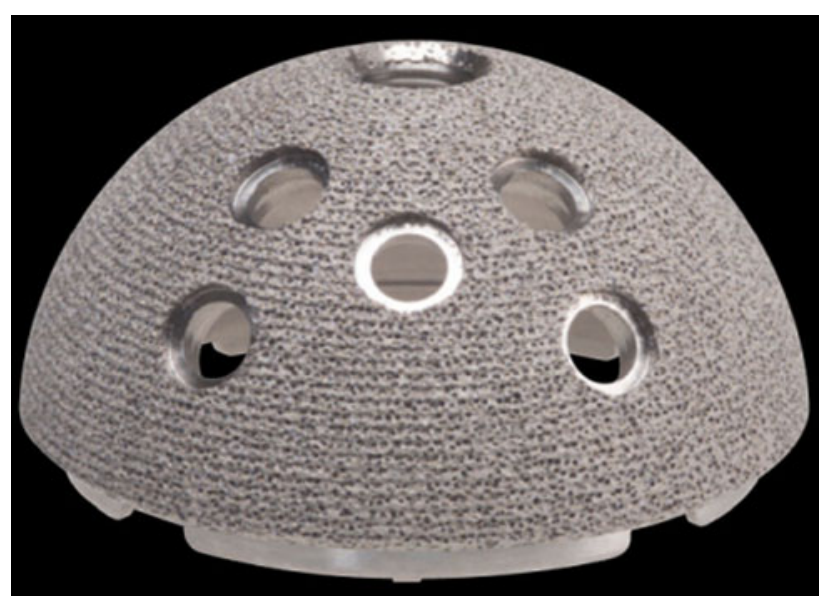

Fig. 14. Tritanium primary acetabular shell, offered in outer diameter sizes ranging from 44 to $66 \mathrm{~mm}$ in diameter.

conventionally machined to a prescribed form and, if desired, mated with a solid Ti alloy substrate. The assembly is treated under a condition where the pore former and binder are removed prior to sintering operations that effectively bond the CPTi particles to each other and the underlying solid $\mathrm{Ti}$ substrate. The final implant design configuration can then be machined (Fig. 14).

Like the first process, this alternative process produces a highly porous implant fixation surface but because of the different method employed to produce it, a unique configuration of the porous surface is formed.

The shape of the pore is largely determined by the shape of the sacrificial particles and how they are dispersed among the particles of CPTi that once sintered together make up the final structure. The magnified images below reveal the typical appearance of the formed pores, that being elliptical in shape and displaying a directionality whereby the long axis of the major pores are oriented parallel to the underlying Ti alloy substrate (Figs. 15, 16). 


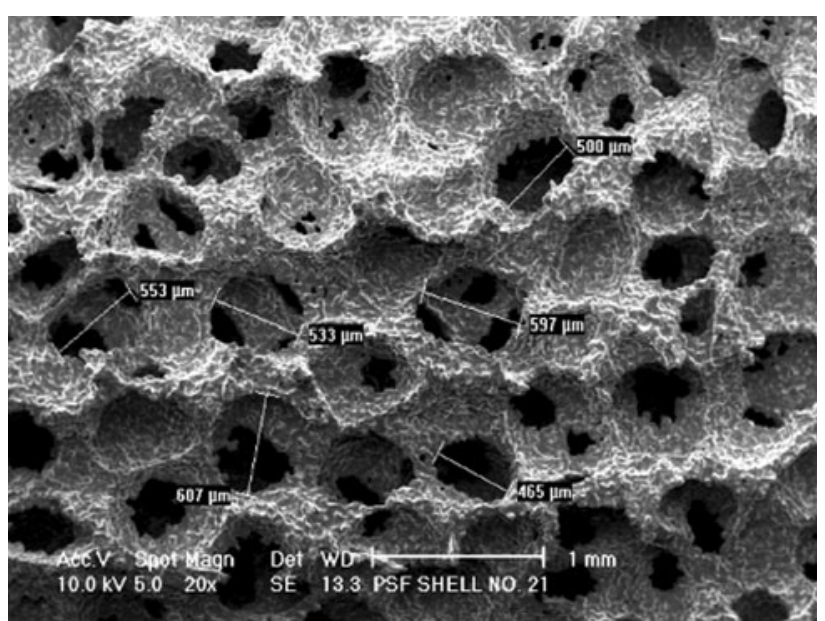

Fig. 15. SEM view of a Tritanium coated shell showing the typical structure of the pores.

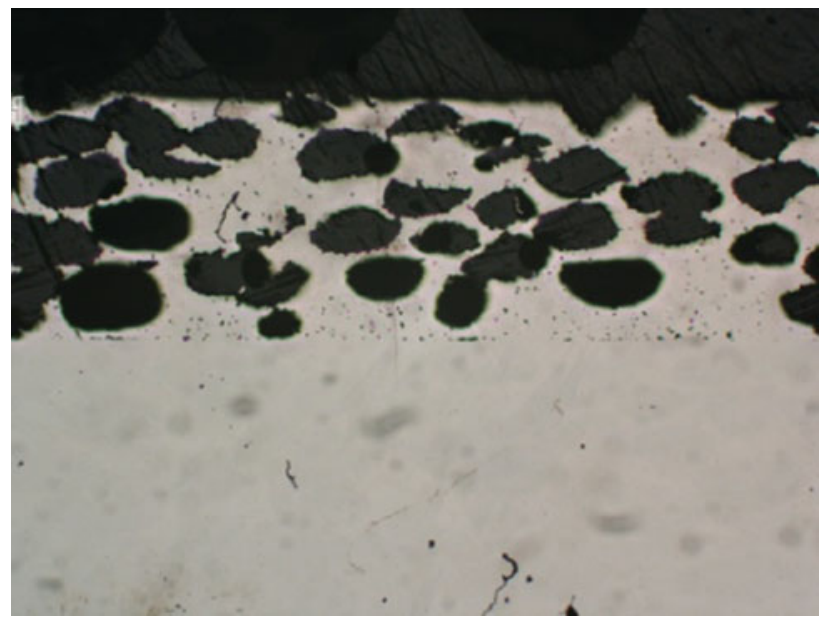

Fig. 16. Cross-sectional light microscopy view of the Tritanium porous surface showing the preferential orientation of the major pores, $\times 15$ magnification.

Major and minor pores across the component size range average $546 \mu \mathrm{m}$ and $311 \mu \mathrm{m}$, respectively $(\times 20$ magnification). In the case of this pore morphology, it is the width of the solid Ti cell walls and their arched configuration that provides the structural integrity of this networked porous architecture. The coating depth and degree of porosity can be determined using the techniques cited earlier (Fig. 17).

In addition to image analysis characterization, a battery of tests is carried out to characterize the chemical and mechanical properties of these highly porous surfaces and the solid substrate materials to which they are applied, including the following:

- Metallurgical analysis

- Microstructure of the modified surface

- Physical properties of the untreated surface

- Mechanical properties of the modified surface

- Mechanical properties of the substrate

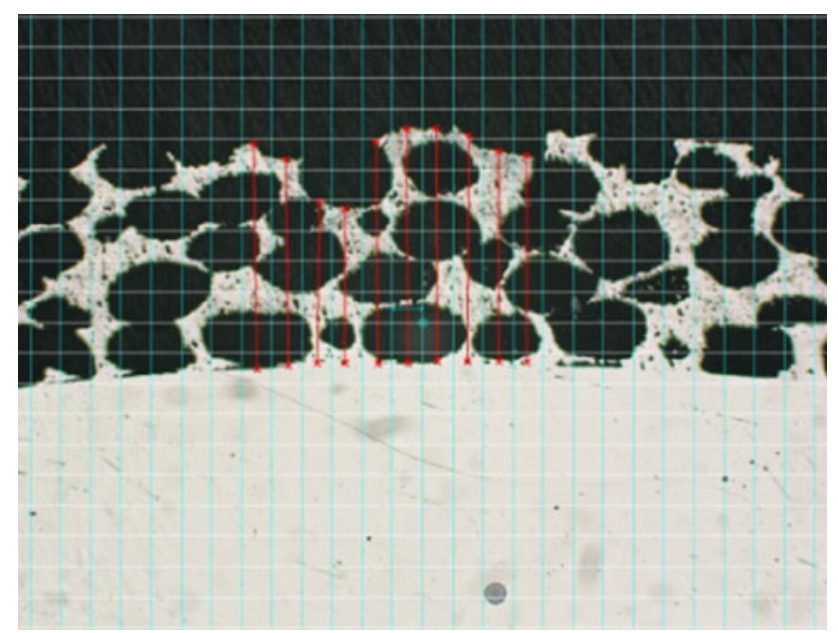

Fig. 17. Depth of Tritanium porous surface, in the range of $1.40 \mathrm{~mm} \pm 0.15 \mathrm{~mm}$. Porosity is approximately $70 \%$ across the component size range, $\times 15$ magnification.

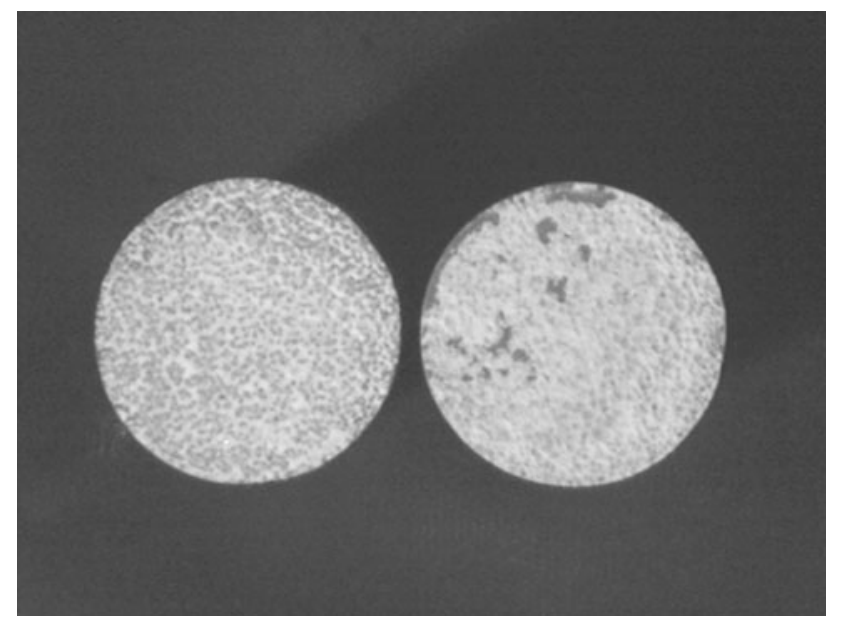

Fig. 18. A typical cohesive coating failure mode for tensile specimens, whereby, failure occurs through the porous struts rather than at the porous surface to substrate interface (The white material is infiltrated epoxy that is used as part of the test method).

Of particular importance to the intended function of these porous surfaces is the strength of the bond that is made with the underlying substrate. Physiologic stresses will be transferred through the interface of the implant and host bone, and it is paramount that the porous surface remain simultaneously fixed to the implant substrate and the biologic tissues that infiltrate the porous network. Testing is conducted to characterize the static tensile, static shear, and shear fatigue strength of the porous surface to substrate interface using coupons that are representative of the actual implant porous coating (Fig. 18).

A U.S. Food and Drug Administration guidance document recommends minimum performance criteria of $20 \mathrm{MPa}$ tensile strength. ${ }^{13}$ Tritanium coatings produced in the manners described typically 

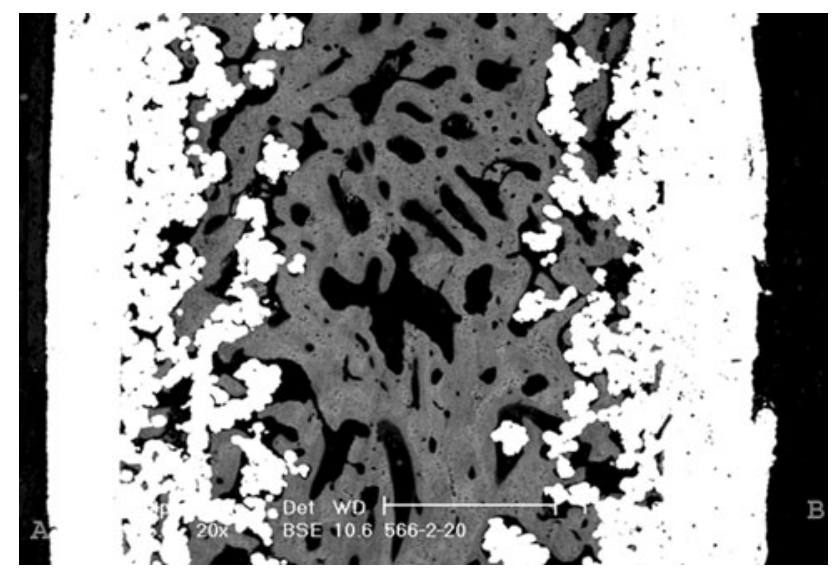

Fig. 19. Scanning electron micrograph of bone penetration into Tritanium channel at 12 weeks. Bone fills the channel and is in intimate contact with titanium throughout the porous structure, $\times 20$ magnification.

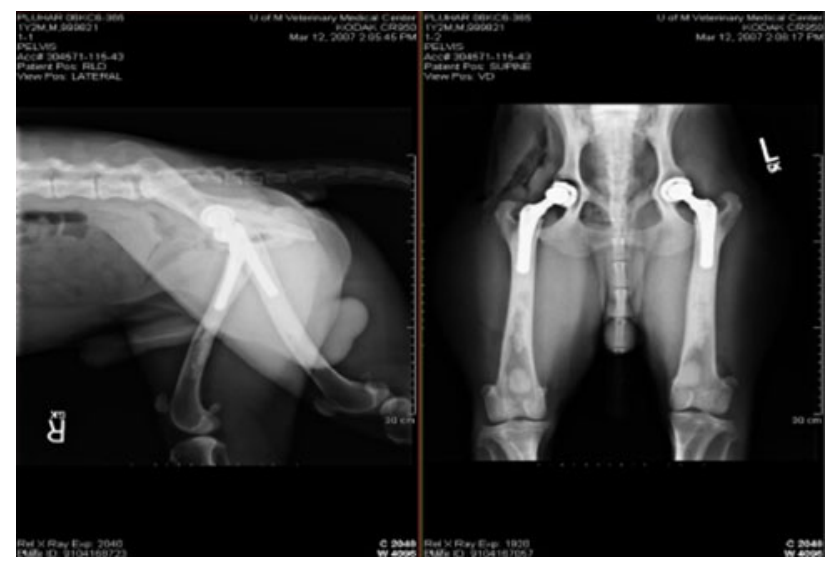

Fig. 20. X-ray of bilateral total hip prostheses implanted in a canine.

display a tensile bond strength near $60 \mathrm{MPa}$ well in excess of the recommended minimum requirement.

In addition to bench top testing, pre-clinical data is often generated to demonstrate safety of devices that employ new materials or performance characteristics. During the development of the Stryker Tritanium porous surfaces several studies in animals were carried out.

In one study that used an implant configuration that allowed a direct comparison of multiple porous material types, bone was shown to fill a channel between opposed plates of porous $\mathrm{Ti}$ and to be in intimate contact throughout the porous structure. The authors reported a far greater amount of bone ingrowth and mechanical strength with the Tritanium highly porous titanium over the other two traditional porous surfaces ${ }^{14}$ (Fig. 19).

In a later study that used a canine total hip implant component, bone ingrowth was seen throughout the porous surface that was present on the canine-sized acetabular component ${ }^{15}$ (Figs. 20, 21 , and 22).

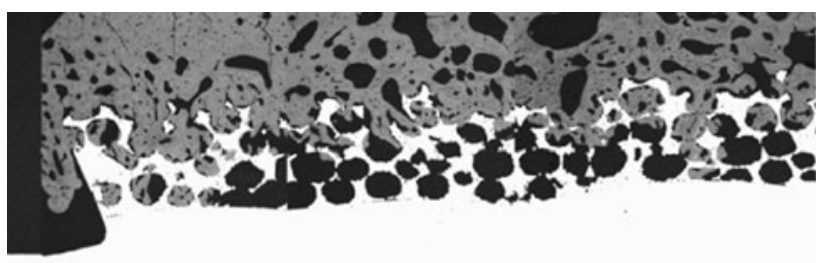

Fig. 21. Bone tissue (gray) infiltrating the porous surface (white) of the acetabular implant component, $\times 20$ magnification.

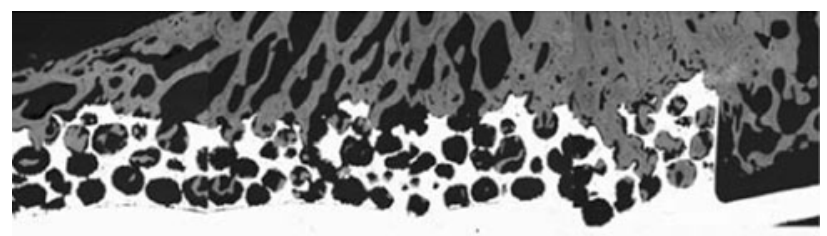

Fig. 22. In the specimens where the metal (white) was in direct contact with bone, cancellous bone (gray) was found within the porous space of the implants and the newly formed bone was continuous with the surrounding bone, $\times 20$ magnification.

Human implants that use these fixation surfaces are currently available and are the subject of ongoing clinical studies.

\section{SUMMARY}

Improved implant fixation strategies with modern highly porous metal technology may well have contributed to the success of hip and knee replacement through enhanced osseointegration. This may lead to greater longevity of implants in patients and better functional outcomes. Continued advances in the technology and manufacturing processes of porous metals will enable improvements in hip and knee replacement fixation and patient outcomes.

\section{OPEN ACCESS}

This article is distributed under the terms of the Creative Commons Attribution License which permits any use, distribution, and reproduction in any medium, provided the original author(s) and the source are credited.

\section{REFERENCES}

1. J.D. Bobyn, R.M. Pilliar, H.U. Cameron, G.C. Watherly, and G.M. Kent, Clin. Orthop. Relat. Res. 149, 291 (1980).

2. R.M. Pilliar, Orthop. Rev. IX, 85 (1980).

3. J.D. Bobyn, R.A. Poggie, J.J Krygier, D.G. Lewallen, A.D. Hanssen, R.J. Lewis, A.S. Unger, T.J. O'Keefe, M.J. Christie, S. Nasser, J.E. Wood, S.D. Stulberg, and M. Tanzer, J. Bone Joint Surg. 86-A, 123 (2004).

4. D.S. Hungerford and R.V. Kenna, Clin. Othop. Relat. Res. 176, 95 (1983).

5. J.L. Ricci, J. Kauffman, W. Jaffe, F. Steiner, M. Hawkins, and H. Alexander (Paper presented at the 23rd Annual Meeting of the Society For Biomaterials, New Orleans, LA, 30 April-4 May, 1997).

6. J. Galante, W. Rostoker, R. Lueck, and R. Ray, J. Bone Joint Surg. 53A, 101 (1971).

7. E. Lembert, J. Galante, and W. Rostoker, Clin. Orthop. Relat. Res. 87, 303 (1972). 
8. A.A. Hofmann, J.D. Evanich, R.P. Ferguson, and M.P Camargo, Clin. Orthop. Relat. Res. 388, 85 (2001).

9. http://tmt.zimmer.com/science.aspx; Science, Trabecular Metal, Zimmer Inc., Warsaw, IN.

10. J.D. Bobyn, G.J. Stackpool, S.A. Hacking, M. Tanzer, and J.J. Krygier, J. Bone Joint Surg. 81-B, 907 (1999)

11. Y. Zhang, P.B. Ahn, D.C. Fitzpatrick, A.D. Heiner, R.A. Poggie, and T.D. Brown, J. Musc. Res 3, 245 (1999).

12. R.A. Cohen, Am. J. Orthop. 31, 216 (2002).

13. Guidance Document for Testing Orthopedic Implants with Modified Metallic Surfaces Apposing Bone or Bone Cement,
28 April, 1994, Orthopedic Devices Branch, Division of General and Restorative Devices, Office of Device Evaluation, Center for Devices and Radiological Health, U.S. Food and Drug Administration, http://www.fda.gov/MedicalDevices/ DeviceRegulationandGuidance/GuidanceDocuments/ucm0810 34.htm.

14. S.R. Frenkel, W.L. Jaffe, F. Dimaano, K. Iesaka, and T. Hua, J. Biomed. Mater. Res. 71B, 387 (2004).

15. C. Ngo, R. Zhang, G. Kulesha, M. Hawkins, J. Zitelli, and G.E. Pluhar (Paper presented at the ORS 2009 Annual Meeting). 